\title{
ON HYPOTHESIS TESTING: A SELECTIVE LOOK AT THE LAGRANGE MULTIPLIER, LIKELIHOOD RATIO AND WALD TESTS*
}

\author{
Kenneth Brown**
}

and

Francisco Cribari-Neto**

\section{Resumo}

O presente artigo discute alguns aspectos relacionados ao uso dos testes multiplicador de Lagrange, razão de verossimilhança e Wald em econometria. Em particular, o artigo se concentra em três tópicos: (i) o teste Wald com restriçōes não-lineares; (ii) conflito entre critérios e correção de tamanho e (iii) derivação de uma estatistica $L M$ melhorada. Resultados de simulação de Monte Carlo ilustram a discussão.

\begin{abstract}
This paper discusses some issues related to the use of the Lagrange multiplier, likelihood ratio and Wald tests in econometrics. In particular, the paper focuses on three issues: (i) the Wald test with nonlinear constraints; (ii) conflict among criteria and size correction; and (iii) the derivation of an improved $L M$ statistic. Some Monte Carlo simulation results illustrate the discussion.
\end{abstract}

\section{Introduction.}

Hypothesis testing is an issue of central concern in econometrics since it can lead to the acceptance or rejection of important theories and hypotheses. Among several, three tests have received much attention in the literature, namely: the Lagrange multiplier, likelihood ratio and Wald tests. These tests are asymptotically equivalent and have the same asymptotic chi-squared distribution. The choice of one of them usually depends on the computational simplicity in

*The insightful comments and suggestions of Silvia L. P. Ferrari are gratefully acknowledged. We also thank Roger Koenker and Chung-Ming Kuan for helpful discussions and two anonymous referees for constructive comments. The usual disclaimers apply.

**From the PhD program in Economics at the University of Illinois.

R. de Econometria Rio de Janeiro v. 12, noㅇ 2, pp.167-194 novembro 1992


the specific context. However, it has been shown that there are several caveats involved in the use of these tests. This paper's aim is to present and discuss some of these caveats. This is accomplished by providing a unified framework to aspects of hypothesis testing that have been treated separately in the literature, and illustrating them with Monte Carlo simulation experiments. The importance of these simulation results stems from the fact that the distribution of the three test statistics considered here is obtained by asymptotic approximations, and it is important to study their performance in finite samples. For general treatments of hypothesis testing, the reader is referred to Buse (1982), Engle (1984), Godfrey (1988) and the references therein.

The paper is organized as follows. The next section presents the three tests and compares their different underlying approaches. Section 3 deals with the issue of using the Wald statistic to test nonlinear hypotheses. It is shown that these tests are sensitive to the specification of the null hypothesis. A simulation experiment compares the performance of the Wald test with and without the Phillips-Park (1988) correction. Section 4 turns to the problem of conflict among criteria. It is shown that there is a systematic relationship which holds among the three test statistics. This means that there exists a level of significance for which the asymptotically equivalent tests lead to different conclusions. It is shown that this problem can be minimized by using size-corrected test statistics. In Section 5 we pursue the issue of deriving an improvement for the Lagrange multiplier statistic. A recent application to a test of heteroskedasticity is also discussed. Finally, some concluding remarks are given in the last section.

\section{Definitions and intuition.}

Given the assumptions of the classical regression model, hypothesis testing can be carried out by application of $t, F$ and $\chi^{2}$ tests from basic statistics courses. However, when the assumption that the error terms are normally distributed breaks down, these statistics no longer follow their respective distributions. In order to be able to carry out tests under these conditions, one must appeal to large sample tests 
such as the Wald, Lagrange multiplier and likelihood ratio tests.

Our setup follows the discussion of Engle (1984). We begin by assuming $y$ is a $T \times 1$ random vector drawn from the joint density $f(\theta, y)$ where $\theta$ is a $K \times 1$ vector of unknown parameters and $\theta \in \Theta$. We will then be interested in testing the hypothesis

$$
\begin{aligned}
& \mathrm{H}_{0}: \theta \in \Theta_{0} \\
& \mathrm{H}_{1}: \theta \in \Theta_{1}
\end{aligned}
$$

where $\Theta_{0} \cap \Theta_{1}=\emptyset$. For example, we might want to test

$$
\begin{aligned}
& \mathrm{H}_{0}: \theta=\theta^{0} \\
& \mathrm{H}_{1}: \theta \neq \theta^{0}
\end{aligned}
$$

with $\theta^{0}$ some known vector of constants. We define the log-likelihood to be

$$
L(\theta, y)=\log f(\theta, y)
$$

which will be maximized at $\hat{\theta}$ satisfying $s(\hat{\theta}, y)=0$, where $s(\cdot)$ is the usual score function. In addition, the asymptotic covariance matrix of $\hat{\theta}$ can be obtained as the inverse of Fisher's Information, or

$$
V(\hat{\theta})=\mathcal{F}^{-1}(\theta) / T
$$

where

$$
\mathcal{F}(\theta)=-\mathrm{E}\left[\frac{\partial^{2} L(\theta, y)}{\partial \theta \partial \theta^{\prime}}\right] / T .
$$

We begin with the Wald test which compares the value of $\theta$ specified in the null hypothesis with the value which maximizes $L(\theta, y)$. If this difference is large, this will be considered evidence that $\mathrm{H}_{0}$ may be false. The statistic is

$$
W=T\left(\hat{\theta}-\theta^{0}\right)^{\prime} \mathcal{F}(\hat{\theta})\left(\hat{\theta}-\theta^{0}\right)
$$

and will have a limiting $\chi^{2}$ distribution with $q$ degrees of freedom, where $q$ is the number of restrictions imposed by $\mathrm{H}_{0}$. Based upon 
Wald (1943), this is the asymptotic approximation to the familiar $t$ and $F$ tests in econometrics.

Next we consider the likelihood ratio test which compares the value of the likelihood function at the maximum, $\hat{\theta}$, and at that specified in the null hypothesis, $\theta^{0}$. If there is a large difference in these values, this is evidence against the null. The statistic is

$$
L R=-2\left(L\left(\theta^{0}, y\right)-L(\hat{\theta}, y)\right)
$$

which again has limiting $\chi^{2}$ distribution with $q$ degrees of freedom.

Finally, we consider the Lagrange multiplier test, also known as Rao's score test (Rao, 1947). This test is derived by maximizing the likelihood function subject to the constraints in $\mathrm{H}_{0}$. If $\mathcal{L}$ is the Lagrangian,

$$
\mathcal{L}=L(\theta, y)-\lambda^{\prime}\left(\theta-\theta^{0}\right),
$$

and $\lambda=s\left(\theta^{0}, y\right)$ from the first order conditions. If we can apply a central limit theorem to the scores, then

$$
L M=s\left(\theta^{0}, y\right)^{\prime} \mathcal{F}^{-1}\left(\theta^{0}\right) s\left(\theta^{0}, y\right) / T
$$

will also have a limiting $\chi^{2}$ distribution with $q$ degrees of freedom. Note that $s\left(\theta^{0}, y\right)$ may be replaced by $\lambda$ in the $L M$ statistic. The idea behind this test is that if the "cost" of imposing the constraint is high, $\lambda$ will be large, and this will imply that the constrained model does not fit the data well. Large values of $\lambda$ will provide evidence against $\mathrm{H}_{0}$.

A visual description of these tests is helpful. If we are simply interested in testing a single parameter, we might obtain a picture similar to Figure 1 . Here, it can be seen that $W$ considers the horizontal distance $\hat{\theta}-\theta^{0}$, while $L R$ examines the vertical distance $L(\hat{\theta}, y)-L\left(\theta^{0}, y\right)$. It can also be seen that $\hat{\theta}-\theta^{0}$ can take equivalent values in two different data sets, with one set closer to the maximum in terms of log-likelihoods. It is for this reason that $W$ is weighted by the inverse of the variance of $\theta$, so that a lower variance will result in a larger value of $W$. The $L M$ test tests the slope of $L(\theta, y)$ evaluated at $\theta^{0}$. Notice that the closer $\theta^{0}$ is to the maximum, the smaller will 


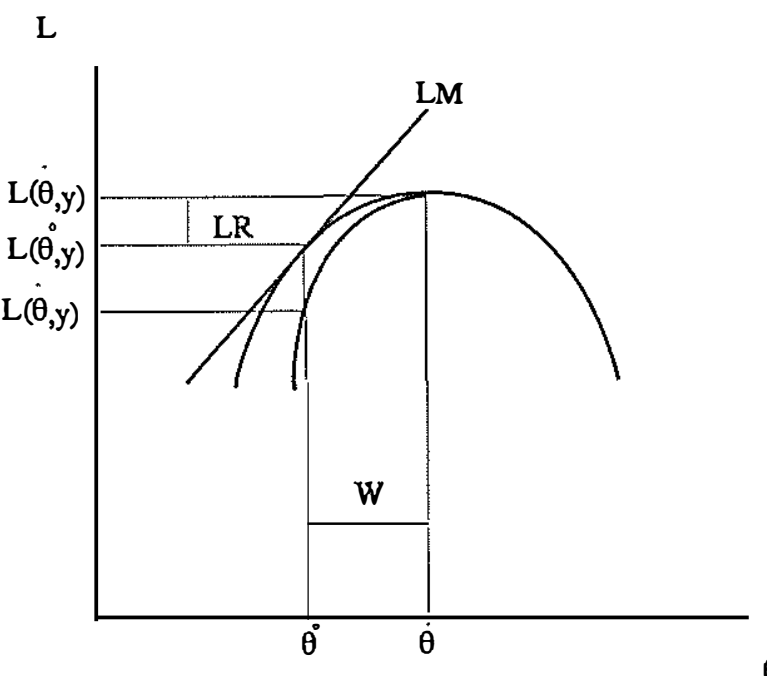

Figure 1.

be the slope in absolute value. Thus, small values of $\lambda$ imply $\theta^{0}$ is "close" to the maximum. Note, though, that "closeness" depends crucially on the shape of the likelihood function. Figure 2 illustrates how two data sets can yield equal values of $\lambda$, but with $\theta^{0}$ closer to the maximum in one case than in the other. It is for this reason that $\mathcal{F}^{-1}\left(\theta^{0}\right) / T$ appears in the $L M$ statistic so that a larger variance results in a larger value of $L M$. A similar graphical exposition of all three statistics can be found in Buse (1982).

Under certain conditions it can be shown that the three test statistics are identical. For example, Engle (1984) provides a proof of the following lemmạ.

Lemma 1. If $L=b-\frac{1}{2}(\theta-\hat{\theta})^{\prime} A(\theta-\hat{\theta})$ where $A$ is a symmetric positive definite matrix which may depend upon the data and upon known parameters, $b$ is a scalar and $\hat{\theta}$ is a function of the data, then $W, L R$ and LM are identical.

Proof: The proof follows directly from (4), (5) and (7). 


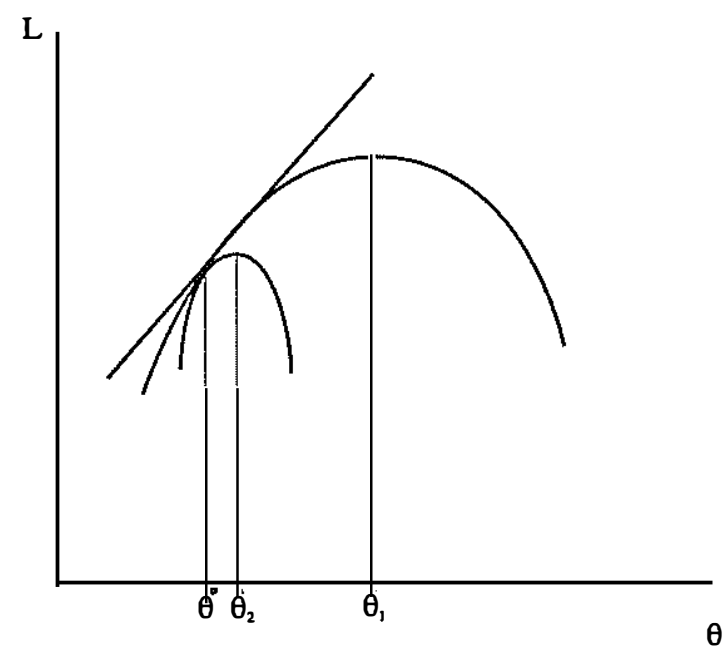

Figure 2.

It can be shown under more general conditions that these three statistics have the same limiting distribution (see Engle (1984) or White (1984)). The essential idea is that when the sample size gets large, the three statistics converge in probability to the same value. The relationship among these statistics is even stronger; since they differ by $O_{p}\left(T^{-1 / 2}\right)$ they are, to first order, the same statistic (Cox, 1988 , p.322). However, as will be noted later, there is a systematic relationship among the three statistics in finite samples. More details on the tests discussed above can be found in Godfrey (1988). For tests of restrictions in the form of inequalities in the linear regression model the reader is referred to Wolak (1987).

\section{The Wald test and nonlinear hypotheses.}

Occasionally in econometrics one needs to test some nonlinear hypotheses. However, it has been shown that algebraically equivalent hypotheses can lead to quite different values of the Wald statistic. See for example Gregory and Veall (1985) or Lafontaine and White 
(1986). In fact, Lafontaine and White showed through Monte Carlo simulation that an investigator can obtain essentially any value of the Wald statistic he chooses by simply "increasing" the nonlinearity. This last point has been proven by Breusch and Schmidt (1988) (see Lafontaine and White, 1986). The Lagrange multiplier and likelihood ratio tests do not suffer from this drawback.

This result is actually quite troublesome since, in general, the Wald test is the most convenient when the unrestricted model is the easiest to estimate. This follows since the $L R$ test requires estimation of both the restricted and unrestricted models for computation and the $L M$ test requires the estimation of the restricted model. One alternative which has been suggested is the implementation of the generalized $c(\alpha)$ test. This test is invariant to the form of the null hypotheses under certain conditions. For a discussion of this test and its invariance properties see Dagenais and Dufour (1991), Neyman (1959) and Smith (1987); see also Dagenais and Dufour (1992) for the problem of lack of invariance of several tests to rescaling.

In the remainder of this section we derive the Wald statistic under various null hypotheses, and then through Monte Carlo simulation we illustrate the problems which can arise. As our example, we choose the simple linear model

$$
y_{t}=\alpha+\beta x_{t}+e_{t},
$$

where $e_{t} \sim \operatorname{NID}(0,1)$. We want to test the hypothesis

$$
\begin{aligned}
& \mathrm{H}_{0}: \beta^{k}=1 \\
& \mathrm{H}_{1}: \beta^{k} \neq 1 .
\end{aligned}
$$

To do this, we generated 5000 samples with sample size $T=20$ and calculated the Wald statistic in each case. The test statistic for this case is given by

$$
W=\frac{\left(\hat{\beta}^{k}-1\right)^{2}}{\left(k \hat{\beta}^{k-1}\right)^{2} V(\hat{\beta})}
$$

for any nonzero exponent $k$ where $V(\hat{\beta})$ is the estimated variance of $\hat{\beta}$ and $\hat{\beta}$ is the MLE (Lafontaine and White, p.36). The independent 
variable was simulated from a uniform distribution on the interval ranging from 10 to 20 and $\alpha$ was taken to be 3 . The simulation results are given in Table 1.

The table presents the percentage of rejections of the null hypothesis in 5000 replications at both the $1 \%$ and $5 \%$ significance levels. Notice, as Lafontaine and White have also shown, that increasing the nonlinearity (by increasing $|k|$ ) leads to an absolute increase in the number of rejections. For $T=17$, these authors estimated the $5 \%$ critical values for different values of $|k|$. Their estimates for $\beta^{40}$ and $\beta^{-40}$ are $335,961,100$ and 7,027,783 respectively (Lafontaine and White, p.37). Obviously this is quite a discrepancy from the value of 3.84 that is used when performing the test.

Table 1.

Empirical size of the uncorrected test, $T=20$

\begin{tabular}{lcc}
\hline Null & Rejections at $5 \%$ & Rejections at $1 \%$ \\
\hline$\beta^{60}$ & $30.70 \%$ & $28.82 \%$ \\
$\beta^{40}$ & $29.70 \%$ & $27.28 \%$ \\
$\beta^{20}$ & $18.06 \%$ & $14.44 \%$ \\
$\beta^{5}$ & $8.26 \%$ & $3.98 \%$ \\
$\beta^{2}$ & $6.82 \%$ & $1.92 \%$ \\
$\beta$ & $6.10 \%$ & $1.70 \%$ \\
$\beta^{-2}$ & $6.32 \%$ & $2.64 \%$ \\
$\beta^{-5}$ & $9.08 \%$ & $5.24 \%$ \\
$\beta^{-20}$ & $18.22 \%$ & $14.66 \%$ \\
$\beta^{-40}$ & $26.34 \%$ & $23.86 \%$ \\
$\beta^{-60}$ & $30.42 \%$ & $28.82 \%$ \\
\hline
\end{tabular}

Although the simulations are not performed here, Lafontaine and White have also shown that the rejection level is quite sensitive to $T$, the sample size (for $T=170$, they estimated the $5 \%$ critical values for $\beta^{40}$ and $\beta^{-40}$ as 91.7 and 126.4 respectively).

Phillips and Park (1988) derived, via an Edgeworth expansion of the null distribution of the Wald statistic, ${ }^{1}$ a correction to the critical

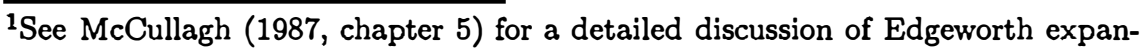


values, correct to $O\left(T^{-1}\right)$, to be used with Wald tests of nonlinear restrictions. $^{2}$ An important assumption in their analysis is that the limiting covariance matrix of $\sqrt{T}(\hat{\psi}-\psi)$ equals the identity matrix, where $\psi=(\alpha, \beta)^{\prime}$. In their paper, they derived the corrected critical values for the test above. These critical values are approximated by

$$
w_{\alpha}^{*}=w_{\alpha}+T^{-1} c\left(w_{\alpha}\right)
$$

where $w_{\alpha}^{*}$ is the corrected critical value for a test of size $\bar{\alpha}, w_{\alpha}$ is the critical value of the $\chi_{q}^{2}$ distribution for size $\bar{\alpha}, T$ is the sample size and

$$
c\left(w_{\alpha}\right)=\sum_{n=0}^{3} \alpha_{n} w_{\alpha}^{n},
$$

where

$$
\begin{aligned}
& \alpha_{0}=0 \\
& \alpha_{1}=0 \\
& \alpha_{2}=-\frac{2}{3}(k-1)(k-2) \\
& \alpha_{3}=\frac{1}{4}(k-1)^{2} .
\end{aligned}
$$

Note that these $\alpha_{n}$ are specific to this example. The more general formulas can be found in Phillips and Park (1988), and the reader is referred to their paper for a complete explanation of this correction. With this correction at hand, we perform simulations for all forms of the test presented in Table 1. The corrected critical values and the simulation results are given in Table 2.

When comparing Tables 1 and 2, it is clear that the test is closer to the nominal size when the corrected critical values are used, even though the limiting covariance matrix of $\sqrt{T}(\hat{\psi}-\psi)$ is not the identity matrix. The sample size used in this simulation was 20 , and we expect that the test would perform much better as the sample size increased.

sions. See also Barndorff-Nielsen and Cox (1989) and Reid $(1988,1991)$.

2 An asymptotic expansion of the null distribution of the Wald statistic for simple hypotheses was previously obtained by Hayakawa and Puri (1985). 
Table 2.

Empirical size of the corrected test, $T=20$

\begin{tabular}{lrrrc}
\hline Null & $w_{05}^{*}$ & \multicolumn{1}{c}{$w_{\text {Nl }}^{*}$} & Rejections at $5 \%$ & Rejections at 1\% \\
\hline$\beta^{60}$ & 785.67 & 7673.65 & $14.84 \%$ & $9.82 \%$ \\
$\beta^{40}$ & 351.95 & 3376.05 & $9.20 \%$ & $4.56 \%$ \\
$\beta^{20}$ & 91.25 & 820.62 & $6.64 \%$ & $2.52 \%$ \\
$\beta^{5}$ & 9.27 & 47.33 & $2.84 \%$ & $0.04 \%$ \\
$\beta^{2}$ & 4.55 & 10.27 & $5.56 \%$ & $0.90 \%$ \\
$\beta$ & 3.84 & 6.63 & $6.10 \%$ & $1.70 \%$ \\
$\beta^{-2}$ & 4.31 & 21.83 & $5.84 \%$ & $0.22 \%$ \\
$\beta^{-5}$ & 8.68 & 76.24 & $3.24 \%$ & $0.02 \%$ \\
$\beta^{-20}$ & 88.89 & 936.23 & $6.02 \%$ & $1.12 \%$ \\
$\beta^{-40}$ & 347.24 & 3607.27 & $8.24 \%$ & $3.48 \%$ \\
$\beta^{-60}$ & 778.59 & 8020.47 & $13.74 \%$ & $8.66 \%$ \\
\hline
\end{tabular}

Notice that the empirical size based on the corrected critical values is still quite large for high values of $|k|$. This implies that there is still room for improvement with this test. To see if the corrected critical values perform better with a larger sample size, we increased the sample size to 100 and estimated the empirical size of the test for all values of $k$ given in Tables 1 and 2, again using 5000 replications. The results are given in Table 3.

For high degrees of nonlinearity, the corrected critical values yield a test much closer to the nominal sizes of $5 \%$ and $1 \%$; however, for values of $|k|$ such as 20 and 5 the corrected test underestimates the nominal size. It appears that the corrected test has stabilized near $5 \%$ and will not "blow up" as the uncorrected test did, but it would be interesting to determine the cause of this oscillating behavior of the corrected test and to derive an improvement to the test to remove this behavior. The results for the $5 \%$ nominal level are displayed in Figure 3.

One question which arises from this situation is - Is any test uniformly most powerful? This question is addressed by means of simulation. The figures in Table 4 are the power of the test for different values of the exponent with data generated under the alternative hypothesis of $\beta=.94, .95, \ldots, 1.06$, and for a sample size of 100 
Table 3.

Empirical sizes of the two tests, $T=100$

\begin{tabular}{rrrrr}
\hline & \multicolumn{2}{c|}{ Corrected } & \multicolumn{2}{c}{ Uncorrected } \\
\hline $\mathrm{k}$ & $5 \%$ & $1 \%$ & \multicolumn{1}{c}{$5 \%$} & $1 \%$ \\
\hline 60 & $5.84 \%$ & $2.04 \%$ & $21.84 \%$ & $18.56 \%$ \\
40 & $3.62 \%$ & $0.70 \%$ & $16.54 \%$ & $13.54 \%$ \\
20 & $1.94 \%$ & $0.10 \%$ & $10.60 \%$ & $6.98 \%$ \\
5 & $3.18 \%$ & $0.24 \%$ & $5.14 \%$ & $1.64 \%$ \\
2 & $4.68 \%$ & $0.88 \%$ & $5.12 \%$ & $1.18 \%$ \\
1 & $5.10 \%$ & $1.18 \%$ & $5.10 \%$ & $1.18 \%$ \\
-2 & $5.00 \%$ & $0.36 \%$ & $5.20 \%$ & $1.32 \%$ \\
-5 & $3.80 \%$ & $0.02 \%$ & $5.56 \%$ & $2.04 \%$ \\
-20 & $2.06 \%$ & $0.00 \%$ & $10.82 \%$ & $.7 .02 \%$ \\
-40 & $3.36 \%$ & $0.42 \%$ & $16.34 \%$ & $13.32 \%$ \\
-60 & $5.58 \%$ & $1.62 \%$ & $21.00 \%$ & $17.88 \%$ \\
\hline
\end{tabular}

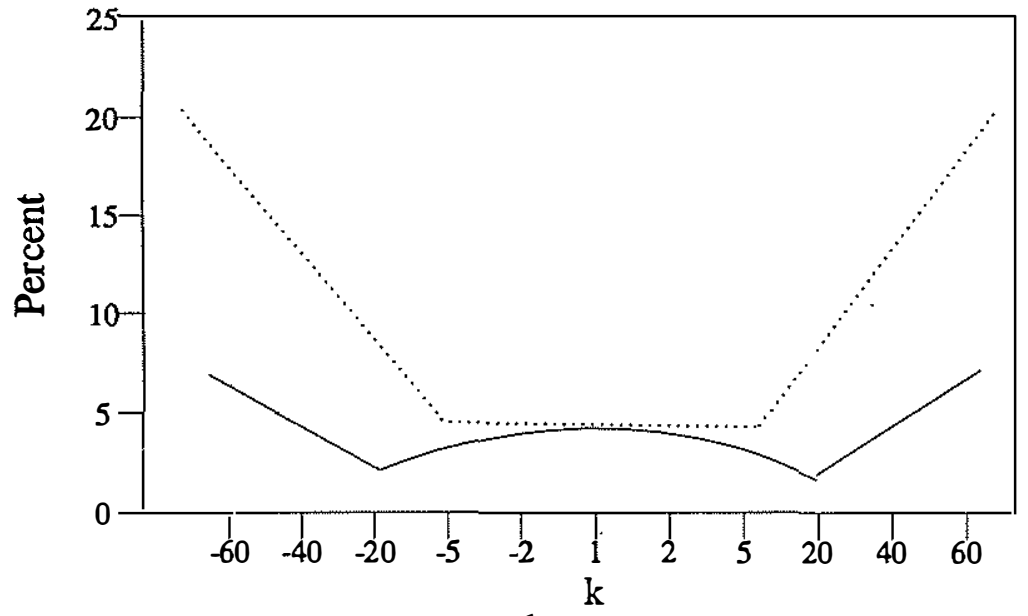

Uncorrected

Corrected

Figure 3. 
Table 4.

Empirical power with estimated critical values, $T=100$

\begin{tabular}{crrrrrrr}
\hline$\beta$ & $k=20$ & $k=5$ & $k=2$ & $k=1$ & $k=-2$ & $k=-5$ & $k=-20$ \\
\hline 0.94 & $52.76 \%$ & $47.62 \%$ & $41.24 \%$ & $39.88 \%$ & $29.36 \%$ & $16.40 \%$ & $0.04 \%$ \\
0.95 & $42.66 \%$ & $37.70 \%$ & $31.02 \%$ & $29.64 \%$ & $20.98 \%$ & $10.98 \%$ & $0.06 \%$ \\
0.96 & $30.70 \%$ & $26.32 \%$ & $20.96 \%$ & $20.16 \%$ & $12.88 \%$ & $6.52 \%$ & $0.38 \%$ \\
0.97 & $22.14 \%$ & $18.62 \%$ & $14.50 \%$ & $13.58 \%$ & $8.32 \%$ & $3.92 \%$ & $0.68 \%$ \\
0.98 & $14.62 \%$ & $12.24 \%$ & $9.38 \%$ & $9.04 \%$ & $6.10 \%$ & $3.10 \%$ & $1.30 \%$ \\
0.99 & $9.34 \%$ & $7.88 \%$ & $6.36 \%$ & $6.08 \%$ & $4.30 \%$ & $3.02 \%$ & $2.24 \%$ \\
1.01 & $2.74 \%$ & $4.40 \%$ & $5.96 \%$ & $7.06 \%$ & $7.84 \%$ & $9.14 \%$ & $9.82 \%$ \\
1.02 & $1.60 \%$ & $5.28 \%$ & $7.84 \%$ & $9.08 \%$ & $11.36 \%$ & $13.54 \%$ & $14.54 \%$ \\
1.03 & $0.72 \%$ & $7.42 \%$ & $11.46 \%$ & $13.34 \%$ & $16.68 \%$ & $20.06 \%$ & $21.48 \%$ \\
1.04 & $0.34 \%$ & $12.74 \%$ & $18.90 \%$ & $21.40 \%$ & $26.02 \%$ & $29.60 \%$ & $31.38 \%$ \\
1.05 & $0.14 \%$ & $17.28 \%$ & $25.74 \%$ & $29.48 \%$ & $34.96 \%$ & $39.76 \%$ & $41.46 \%$ \\
1.06 & $0.06 \%$ & $24.70 \%$ & $33.90 \%$ & $37.92 \%$ & $44.24 \%$ & $49.22 \%$ & $51.24 \%$ \\
\hline
\end{tabular}

observations. The values in the table represent the percentage of rejections of the null hypothesis at the 5 percent level of significance in 5000 replications for various values of $k$, and the test was performed with estimated (empirical) critical values. This was done in order to force the tests to have the same size, since otherwise power comparisons would be meaningless. A graphical presentation of these results is given in Figures 4 and 5. It appears from the table that smaller values of $|k|$ yield a higher power in general. We note, however, that low values of $|k|$ are not uniformly most powerful. Larger or negative values of $k$ result in a higher power, but only to one side. Thus, the small values of $|k|$ ( 1 or 2 ) appear to be best for two sided tests, while the others seem to perform better for one sided tests. In particular, note that in Figure 4 no power curve is always above all others. Although the design of our simulation experiment is somewhat different from the one performed by Lafontaine and White, our results seem to confirm their findings.

Although the Wald test is often performed, it is not clear that the results obtained in finite samples are as accurate as one would like, especially with nonlinear restrictions. Using the corrected critical values of Phillips and Park, the test is closer to achieving the correct size, although still erratic in simulations. 


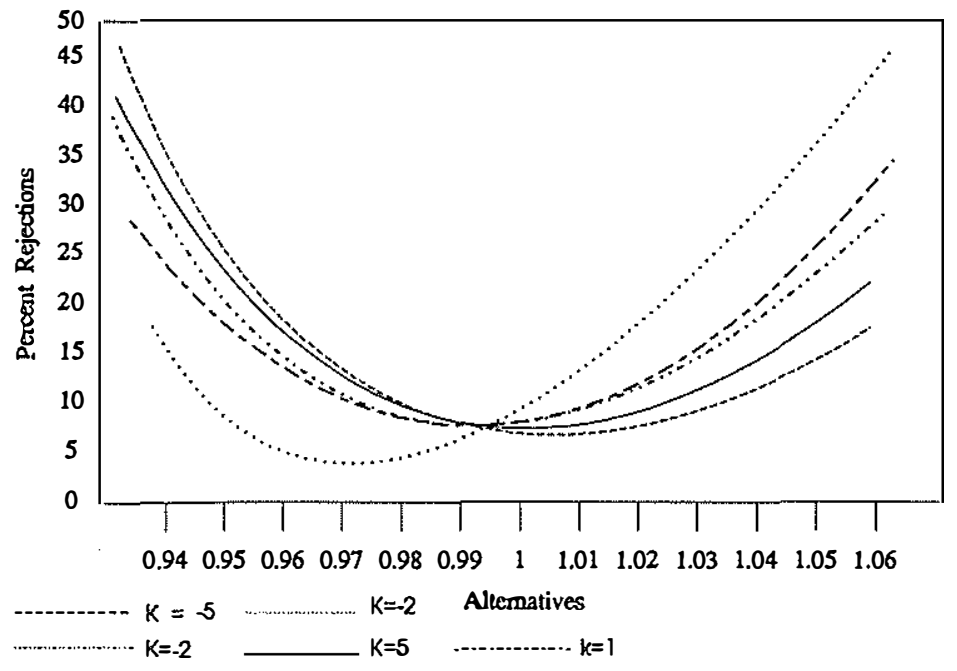

Figure 4.

4. Conflict among criteria and size correction.

As shown before, the Lagrange multiplier, likelihood ratio and Wald statistics are asymptotically equivalent in the sense that the difference between any two of those statistics vanishes in probability as the sample size tends to infinity. Put differently, all three statistics converge in distribution to a chi-squared with the number of degrees of freedom given by the number of restrictions imposed. Hence, the asymptotic critical regions for the three tests are the same.

Nevertheless, in finite samples there is a systematic relationship that holds among the three test statistics. This was first noted by Berndt and Savin (1977) and Savin (1976). A natural implication of such a systematic relationship is that there always exists a level of significance for which the tests lead to different conclusions, that is, there is conflict among the criteria. For instance, Sims (1980, p.155) notes that "asymptotically equivalent formulas for the test statistic 


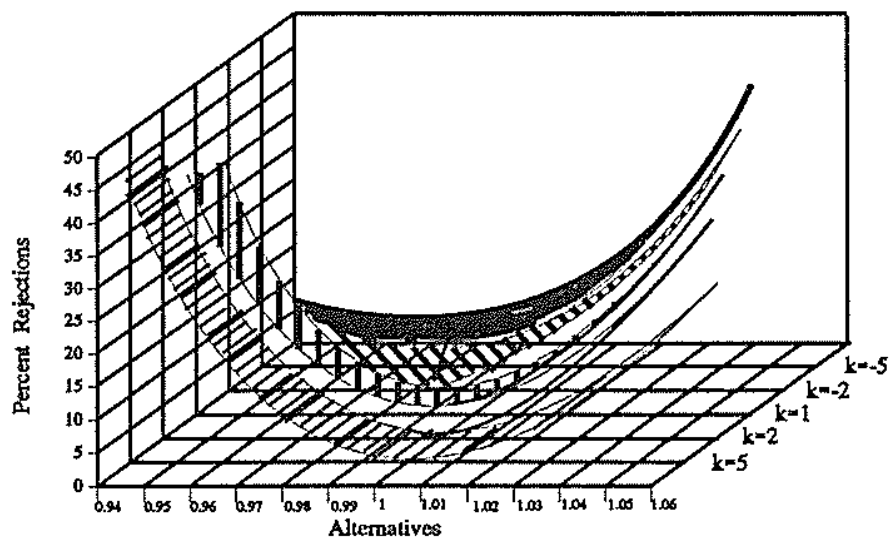

Figure 5.

may give very different significance levels for the same data." Indeed, the simulations in Evans and Savin (1982) show that the probability of conflict can be very large. They find that this probability can be as high as 37 percent. The dispersion, however, will tend to zero as the number of observations increases. The analysis in Berndt and Savin (1977) assumes i.i.d. disturbances. This assumption is relaxed in Breusch (1979), Rothenberg (1984) and Savin (1976).

Following Breusch (1979), consider the following linear model

$$
y=X \beta+\varepsilon,
$$

where $y$ is a $T$-vector of observations of the response, $X$ is a $T \times K$ matrix of covariates, $\beta$ is a $K$-vector of parameters, and $\varepsilon$ is a $T$-vector 
of disturbances with $E(\varepsilon)=0$ and $E\left(\varepsilon \varepsilon^{\prime}\right)=\Lambda$. The hypothesis to be tested is

$$
R \beta=r,
$$

where $R$ is a $q \times K$ matrix $(q \leq K)$ which imposes $q$ linear restrictions on the vector of parameters $\beta$.

The likelihood for the estimation of $\beta$ and $\Lambda$ is given by

$$
L(\beta, \Lambda)=(2 \pi)^{-T / 2}|\Lambda|^{-1 / 2} \exp \left[\frac{-(y-X \beta)^{\prime} \Lambda^{-1}(y-X \beta)}{2}\right] .
$$

We then have the following result:

Lemma 2. Let $\hat{\Lambda}$ be the maximum likelihood (ML) estimate of $\Lambda$, and $\hat{\Lambda}$ be the ML estimate of $\Lambda$ satisfying restriction (14). Assume that the $M L$ estimates of the parameters in $\beta$ are not correlated with those of the parameters in the covariance matrix $\Lambda$. Then,

$$
\begin{gathered}
L M=-2 \log \left[\frac{\sup _{R \beta=r} L(\beta \mid \tilde{\Lambda})}{\sup _{\beta} L(\beta \mid \tilde{\Lambda})}\right], \\
L R=-2 \log \left[\frac{\sup _{R \beta=r, \Lambda} L(\beta, \Lambda)}{\sup _{\beta, \Lambda} L(\beta, \Lambda)}\right],
\end{gathered}
$$

and

$$
W=-2 \log \left[\frac{\sup _{R \beta=r} L(\beta \mid \hat{\Lambda})}{\sup _{\beta} L(\beta \mid \hat{\Lambda})}\right] .
$$

Proof: See Breusch (1979).

We can now state the following theorem:

Theorem 1. Given the conditions in Lemma 2,

$$
W \geq L R \geq L M \text {. }
$$

Proof: The proof follows directly from (16), (17) and (18).

Theorem 1 states that in finite samples there exists a systematic relationship among the three test statistics. Thus, as mentioned before, there always exists a level at which asymptotically equivalent tests for the same hypothesis lead to different conclusions. 
Nonetheless, the relationship in (19) does not imply that the Wald test is more powerful than the others, since this test also has a higher probability of rejection under the null hypothesis. ${ }^{3}$ It is important, therefore, to obtain size-corrected statistics in order to make comparisons and/or reduce the probability of conflict, as, e.g., in Evans and Savin (1982).

Suppose that we have the linear model in (13) and the restriction in (14). Assume, however, that $E\left(\varepsilon \varepsilon^{\prime}\right)=o^{2} I$, i.e., the covariance matrix is scalar. For a slightly more general framework where this assumption is relaxed, see Rothenberg (1984).

Letting $\hat{\beta}$ and $\hat{\sigma}^{2}$ represent the unconstrained ML estimates of $\beta$ and $\sigma^{2}$ respectively, and $\ddot{\beta}$ and $\ddot{\sigma}^{2}$ the corresponding constrained ones, we have that $L R=T\left[\log \ddot{\sigma}^{2}-\log \hat{\sigma}^{2}\right], L M=(R \hat{\beta}-$ $r)^{\prime}\left[\ddot{\sigma}^{2} R\left(X^{\prime} X\right)^{-1} R^{\prime}\right]^{-1}(R \hat{\beta}-r)$ and $W=(R \hat{\beta}-r)^{\prime}\left[\hat{\sigma}^{2} R\left(X^{\prime} X\right)^{-1} R^{\prime}\right]^{-1}$ $(R \hat{\beta}-r)$. It follows from straightforward algebra that:

$$
\begin{gathered}
W=T\left[\frac{R R S S-U R S S}{\zeta R S S}\right], \\
L M=\frac{W}{1+\frac{W}{T}},
\end{gathered}
$$

and

$$
L R=T \log \left[1+\frac{W}{T}\right],
$$

where URSS and RRSS stand for unrestricted residual sum of squares and restricted residual sum of squares, respectively.

It is also clear from (20), (21) and (22) that $W \geq L R \geq L M$ holds. Note also that when $W / T$ is small the following approximation holds: $W-L R \doteq L R-L M$ (Evans and Savin, 1982, p.740; Rothenberg, 1984, p.831).

A useful correction for the three test statistics can be obtained through an Edgeworth expansion. For the likelihood ratio statistic and under the null hypothesis, we just replace $T$ by $[(T-K)+$ $(q / 2)-1]$, which follows from Theorem 8.5.2 in Anderson (1984, p.

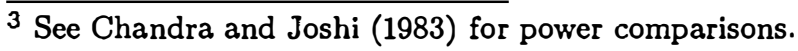


317). ${ }^{4}$ The corrected statistic is then given by

$$
L R^{c}=\gamma \log \left[\frac{R R S S}{U R S S}\right],
$$

where $\gamma=[(T-K)+(q / 2)-1]$. Now, the $L R$ test has the correct significance level to order $\bar{T}^{-1}$, where $\bar{T}=(T-K)$. By making use of the expansion for the $L R$ test statistic, it can be shown that the corrected Lagrange multiplier and Wald statistics (to order $\bar{T}^{-1}$ ) are given by

$$
L M^{c}=\frac{\gamma}{2(\gamma+1)-q}\left\{\log \left[\frac{R R S S}{U R S S}\right]\right\}\left\{2(\gamma+2)-\gamma \log \left[\frac{R R S S}{U R S S}\right]\right\}
$$

and

$$
W^{c}=\frac{\gamma}{2(\gamma+1)-q}\left\{\log \left[\frac{R R S S}{U R S S}\right]\right\}\left\{2(\gamma-q+2)+\gamma \log \left[\frac{R R S S}{U R S S}\right]\right\} .
$$

It should be noted that since the corrections to the Lagrange multiplier and Wald statistics follow from the corrected likelihood ratio, they require the estimation of the model both with and without the specified restrictions, which is an undesirable property.

The size-corrected tests have approximately the right significance level, and the probability of conflict in practical situations is now negligible.

The discussion above relates to the linear model with normal errors. It should be clear that when one relaxes the assumption of normality the relationship in (19) does not necessarily hold. Ullah and Zinde-Walsh (1984) investigated the relationship between the three test statistics in the linear model with errors distributed according to a multivariate Student $t$; see also Ullah and Zinde-Walsh (1985). In this framework and assuming that $W / T$ is small, they have shown that

$$
W(t) \geq \lambda L R(t) \geq \lambda^{2} L M(t)
$$

${ }^{4}$ See also Italianer (1985). For approximations to the probability density function of the likelihood ratio statistic under both the null and non-null cases, see Nagar and Chandrika (1992). 
where $\lambda=(T+\zeta) /(T+\zeta+2), \zeta$ is the degrees of freedom parameter of the multivariate Student $t$ distribution, and the $(t)$ after each statistic signifies that such a statistic was obtained based on a Student $t$. Since $0<\lambda<1$, the relationship in (26) is weaker than the one obtained under the assumption of normality and given in (19).

Also, note that the relationship in (19) was obtained for linear restrictions. Suppose we are interested in testing a nonlinear restriction in the context of the normal linear model. It then follows from the analysis in Breusch (1979) that even in this case $L R \geq L M$. However, this accounts only for part of the relationship in (19). The simulation results for this case in Mizon and Hendry (1980) show that there are situations where $L R>W$, which violates (19).

Finally, for the case of testing nonlinear restrictions in the normal linear model, Mizon (1977) found some cases where $L R>W$, again violating (19).

\section{An improved Lagrange multiplier statistic.}

In the last section, an expansion for the likelihood ratio statistic was obtained, and based on this expansion corrected Lagrange multiplier and Wald statistics were obtained. This section will continue to pursue the issue of size correction, but it will focus on the Lagrange multiplier test, since from the three tests considered the $L M$ is the most used in econometrics. This is because, as shown before, it only requires the estimation of the constrained model. However, this section will be concerned with a direct expansion of the Lagrange multiplier statistic distribution function. ${ }^{5}$

An important result in the improvement of the $L M$ statistic was obtained by Harris (1985). Suppose we have a set of $T$ independent observations $y=\left(y_{1}, \ldots, y_{T}\right)^{\prime}$ whose log-likelihood depends on $K$ parameters $\theta=\left(\theta_{1}, \ldots, \theta_{K}\right)$. Consider the following partition: $\theta=\left(\theta_{(1)}^{\prime}, \theta_{(2)}^{\prime}\right)^{\prime}$, where $\theta_{(1)}=\left(\theta_{1}, \ldots, \theta_{K-q}\right)^{\prime}$ and $\theta_{(2)}=$ $\left(\theta_{K-q+1}, \ldots, \theta_{K}\right)^{\prime}$. The hypothesis of interest is $\mathrm{H}_{0}: \theta_{(2)}=\theta_{(2)}^{(0)}$,

${ }^{5}$ The two main results in this section are presented as theorems. However, one has to bear in mind that there are important technical regularity conditions required for the validity of such results. Silvia Ferrari brought this point to our attention. 
against a two-sided alternative. Notice that this includes the case where $R=\left[0: I_{q}\right]$ in the context of our linear regression model in (13). However, it can be used in more general settings, as, for instance, to test whether a parameter (or a set of parameters) of a certain distribution equals a given value. Now let $s(\theta)=\partial L(\theta) / \partial \theta=$ $\left[s_{1}^{\prime}\left(\theta_{(1)}, \theta_{(2)}\right), s_{2}^{\prime}\left(\theta_{(1)}, \theta_{(2)}\right)\right]^{\prime}$. Then, $L M=\ddot{s}_{2}^{\prime} \ddot{B}^{22} \ddot{s}_{2}$, where $B^{22}$ is the asymptotic covariance matrix of $\hat{\theta}_{2}$. Here, " $\ddot{f}$ " indicates that the function $f$ is been evaluated at $\left(\tilde{\theta}_{(1)}^{\prime},\left(\theta_{(2)}^{(0)}\right)^{\prime}\right)^{\prime}$, where $\tilde{\theta}_{(1)}$ denotes the restricted maximum likelihood estimate of $\theta_{(1)}$. Further, let $F_{L M}(z)$ be distribution function of the $L M$ statistic and $G_{q}(z)$ the distribution function of a central chi-squared random variable with $q$ degrees of freedom. Then, Harris' result can be stated as follows:

Theorem 2. The null distribution of the Lagrange multiplier statistic has the following asymptotic expansion to order $T^{-1}$ :

$$
\begin{gathered}
F_{L M}(z)=G_{q}(z)+(24 T)^{-1}\left[\delta_{1} G_{q+6}(z)+\delta_{2} G_{q+4}(z)\right. \\
\left.+\delta_{3} G_{q+2}(z)+\delta_{4} G_{q}(z)\right]
\end{gathered}
$$

where $\delta_{1}=A_{3}, \delta_{2}=\left(A_{2}-3 A_{3}\right), \delta_{3}=\left(3 A_{3}-2 A_{2}+A_{1}\right), \delta_{4}=$ $\left(A_{2}-A_{1}-A_{3}\right)$, and the $A$ 's (given in Harris' (1985) paper) are functions of the joint cumulants of the derivatives of the log-likelihood for the full data.

ProOF: See Harris (1985).

The reader is referred to Harris' paper for a definition of the $A$ 's or to Ferrari and Cordeiro $(1990,1992)$ for matrix expressions for these coefficients. The result above is very useful since it allows one to obtain size-corrected critical values for the Lagrange multiplier test by using Hill and Davis' (1968) inverse expansion formula. Letting $q$ represent the difference in the parameter space under the null and alternative hypotheses, we have that the corrected critical value $\left(a^{*}\right)$ is given by

$$
\begin{aligned}
a^{*}=a & +\frac{1}{12 T}\left\{\frac{a\left[a^{2}+(q+4) a+(q+2)(q+4)\right]}{q(q+2)(q+4)} \delta_{1}\right. \\
& \left.+\frac{a(a+q+2)}{q(q+2)} \delta_{2}^{\prime}+\frac{a}{q} \delta_{3}\right\}
\end{aligned}
$$


where $\operatorname{Pr}\left(\chi_{q}^{2} \geq a\right)=\gamma_{0}$ where $\gamma_{0}$ is the nominal size of the test and $\delta_{2}^{\prime}=A_{2}-3 A_{3}$.

For instance, it was noticed by Cribari-Neto and Ferrari (1992), Godfrey (1978), Griffiths and Surekha (1986) and Honda (1988) that the Lagrange multiplier test of homoskedasticity rejects the null hypothesis less often than expected based on the its nominal size. In particular, Honda (1988) applied Harris' result to get modified critical values for the $L M$ test of homoskedasticity.

Honda's (1988) application of the result in Theorem 2 can be described as follows. Consider the linear regression model given in (13) with the disturbances $\varepsilon_{t}$ distributed independently according to a normal distribution with mean zero and variance $\sigma_{t}^{2}=h\left(w_{t}^{\prime} \alpha\right)$ for $t$ ranging from 1 to $T$, where $w_{t}^{\prime}=\left(1 v_{t}^{\prime}\right)$ is a $1 \times(q+1)$ vector of exogenous variables, $\alpha$ is a $(q+1)$-vector of parameters, and $h(\cdot)$ is a function of unknown form. The homoskedasticity test then has the null $\mathrm{H}_{0}: \alpha_{i}=0 \quad \forall i \geq 2$. Notice that this specification includes both additive and multiplicative heteroskedasticity as special cases. Then, the $L M$ statistic is given by $L M=u^{\prime} W\left(W^{\prime} W\right)^{-1} W^{\prime} u / 2$, where $u$ is a $T$-vector with elements $\left(\hat{\varepsilon}_{t}^{2}-\hat{\sigma}^{2}\right) / \hat{\sigma}^{2}$, and $W$ is a $T \times(q+1)$ matrix such that $W^{\prime}=\left(w_{1}, \ldots, w_{T}\right)$. The size-corrected critical value of the $L M$ test for homoskedasticity is given by (29), and the coefficients ( $A$ 's) are given by

$$
\begin{gathered}
A_{1}=24 q(K-1)-24 T \sum_{t=1}^{T} H_{t} J_{t}+6 T \sum_{t=1}^{T} \sum_{s=1}^{T} H_{t s}\left(J_{t} J_{s}+2 J_{t s}^{2}\right),(30 \mathrm{a}) \\
A_{2}=-24 q^{2}+36 T \sum_{t=1}^{T} H_{t}^{2}-48 \sum_{t=1}^{T} \sum_{s=1}^{T} H_{t s}^{2}-24 T \sum_{t=1}^{T} \sum_{s=1}^{T} H_{t s} H_{s} J_{t} \\
A_{3}=24 T \sum_{t=1}^{T} \sum_{s=1}^{T} H_{t} H_{s} H_{t s}+16 T \sum_{t=1}^{T} \sum_{s=1}^{T} H_{t s}^{3},
\end{gathered}
$$

where $\bar{v}=T^{-1} \sum_{t=1}^{T} v_{t}, M=\sum_{t=1}^{T}\left(v_{t}-\bar{v}\right)\left(v_{t}-\bar{v}\right)^{\prime}, \quad H_{t}=\left(v_{t}-\right.$ $\bar{v})^{\prime} M^{-1}\left(v_{t}-\bar{v}\right), \quad H_{t s}=\left(v_{t}-\bar{v}\right)^{\prime} M^{-1}\left(v_{s}-\bar{v}\right), J_{t}=x_{t}^{\prime}\left(X^{\prime} X\right)^{-1} x_{t}$, $J_{t s}=x_{t}^{\prime}\left(X^{\prime} X\right)^{-1} x_{s}$. Moreover, $\sum_{t=1}^{T} J_{t}=K$ and $\sum_{t=1}^{T} H_{t}=q$. 
Cribari-Neto and Ferrari (1992) give matrix expressions for the $A$ 's given in (30a)-(30c), and the size corrected test can be more easily implemented using their formulas. Honda's simulations show that the $L M$ test with the corrected critical value in (29) performs better than both the standard $L M$ test and Koenker's (1981) version.

A further step in the improvement of the Lagrange multiplier test was taken by Cordeiro and Ferrari (1991). Building upon Harris' work, these authors obtained an improved Lagrange multiplier statistic to order $T^{-1}{ }^{6}$

Recall that Harris did not obtain such a statistic, but only an expansion of the null distribution of the Lagrange multiplier statistic that enables one to obtain improved critical values by using Hill and Davis' (1968) inverse expansion formula. The main result in Cordeiro and Ferrari (1991) can then be stated as follows. ${ }^{?}$

Theorem 3. An improved Lagrange multiplier statistic having $\chi_{q}^{2}$ distribution to order $T^{-1}$ is given by

$$
L M^{*}=L M\left[1-b\left(L M, q, \delta_{1}, \delta_{2}^{\prime}, \delta_{4}\right)\right]
$$

where

$$
b\left(z, q, \delta_{1}, \delta_{2}^{\prime}, \delta_{4}\right)=\frac{\delta_{1}}{12 T q(q+2)(q+4)} z^{2}+\frac{\delta_{2}^{\prime}}{12 T q(q+2)} z-\frac{\delta_{4}}{12 T q} .
$$

Proof: See Cordeiro and Ferrari (1991).

Note that we have divided equation (7) in Cordeiro and Ferrari by $T$ in order to make their result compatible with Harris' notation. Note also that this is not yet a readily applicable result, since the improved Lagrange multiplicr statistic $L M^{*}$ involves unknown parameters. However, one can use maximum likelihood estimates of

\footnotetext{
${ }^{6}$ For improved Lagrange multiplier tests for generalized linear models, see Cordeiro, Ferrari and Paula (1993).

${ }^{7}$ An improved Lagrange multiplier statistic was also obtained by Mukerjee (1992). Here we focus on Cordeiro and Ferrari's (1991) results because they are much less restritive than Mukerjee's.
} 
the parameters necessary to the computation of the $L M$ statistic under the null hypothesis. Such a statistic will have $\chi_{q}^{2}$ distribution to $O\left(T^{-1}\right)$.

A simple Monte Carlo simulation experiment can illustrate the Bartlett-type correction described above. In this simulation exercise we consider the simple case of testing the mean of a normal distribution when the variance is unknown. The Lagrange multiplier statistic for this test is given by

$$
L M=\frac{T^{2}\left(\bar{y}-\mu_{0}\right)^{2}}{\sum_{t=1}^{T}\left(y_{t}-\mu_{0}\right)^{2}},
$$

where $\mu_{0}$ is the value specified for the mean in the null hypothesis and $\bar{y}$ is the sample mean. The improved Lagrange multiplier statistic can be obtained by using the following formula (Cordeiro and Ferrari, p.579)

$$
L M^{*}=L M\left(1-\frac{3-L M}{2 T}\right) .
$$

The simulation experiment was conducted as follows. The two statistics given above were calculated for 5000 replications of a standard normal distribution. The null for the test was taken to be $\mathrm{H}_{0}: \mu=0$, and the nominal size was chosen to be $5 \%$. The results for different sample sizes are displayed in Table 5. The figures in Table 5 show that the corrected $L M$ statistic has an empirical size closer to the nominal size than the uncorrected one. It is clear that the smaller. the sample size the better the Bartlett-type correction works. As the number of observation increases, the empirical size of both statistics approach the nominal size of the test.

A further question in this context is whether the correction discussed above implies any loss of power. We addressed this question by running another Monte Carlo experiment. Again, the nominal size was taken to be $5 \%$, the number of replications was set to 5000 , and the null hypothesis was that the mean of a normal distribution equals zero. The sample size in this experiment was chosen to be 30 , and both statistics were computed for drawings from normal distributions with different means and unit variance. The results are given in Table 6 . It is clear from the results in Table 6 that the 
Table 5.

Empirical sizes of the two tests

\begin{tabular}{rll}
\hline $\mathrm{T}$ & $L M$ & $L M^{*}$ \\
\hline 5 & $1.8 \%$ & $2.7 \%$ \\
10 & $3.9 \%$ & $4.3 \%$ \\
15 & $4.6 \%$ & $5.0 \%$ \\
20 & $4.6 \%$ & $4.9 \%$ \\
25 & $4.5 \%$ & $4.7 \%$ \\
30 & $5.0 \%$ & $5.0 \%$ \\
\hline
\end{tabular}

corrected statistic performs always slightly better than the original one in terms of power. Although this difference does not seem to be significant, the important result is that the Bartlett-type correction to the Lagrange multiplier test statistic does not imply any loss of power.

Table 6 .

Empirical powers of the two tests, $T=30$

\begin{tabular}{crr}
\hline$\mu$ & $L M$ & $L M^{*}$ \\
\hline-0.6 & $88.7 \%$ & $89.0 \%$ \\
-0.4 & $55.1 \%$ & $55.6 \%$ \\
-0.2 & $17.9 \%$ & $18.3 \%$ \\
-0.1 & $7.9 \%$ & $8.0 \%$ \\
0.1 & $7.3 \%$ & $7.6 \%$ \\
0.2 & $18.2 \%$ & $18.5 \%$ \\
0.4 & $55.4 \%$ & $55.8 \%$ \\
0.6 & $88.5 \%$ & $88.8 \%$ \\
\hline
\end{tabular}

Cribari-Neto and Ferrari (1992), building upon the result discussed above, derived a Bartlett-type correction to the Lagrange multiplier test of heteroskedasticity. ${ }^{8}$ Their statistic follows directly from

\footnotetext{
${ }^{8}$ Bartlett corrections for the likelihood ratio test of heteroskedasticity were derived by Attfield (1991) and Cordeiro (1993).
} 
Theorem 3. The $A$ 's are given in (30a)-(30c) or by some matrix formulas given in their paper. An important feature of their analysis is that it shows that for some special models the chi-squared asymptotic approximation to the distribution function of the LM statistic is better or worse depending on the sample measures of skewness and kurtosis of the independent variable. They also show that the Bartlett-corrected test can easily be implemented for some particular models. The simulation results presented by these authors show that both their test and Honda's test perform better than the original Lagrange multiplier test.

Both Harris' and Cordeiro and Ferrari's results are remarkable steps in obtaining an improvement for the Lagrange multiplier test in finite samples. The use of the modifications suggested by these authors can lead to more accuracy in several tests in practical situations and hence to more reliable results.

\section{Conclusion.}

The purpose of this paper was to discuss some selected aspects of three asymptotically equivalent tests: the Lagrange multiplier, likelihood ratio and Wald tests. These tests can lead to different conclusions in finite samples. This is because there are several caveats in the use of such tests. First, it was shown that when testing nonlinear constraints the Wald test is sensitive to the specification of the null hypothesis, and that one can minimize this problem by using the correction proposed by Phillips and Park (1988). Second, we have shown that two econometricians using the same data set and asymptotically equivalent tests can reach different conclusions. However, it is possible to derive size corrections to the test statistics in order to make the probability of conflict among criteria negligible in practical situations. Finally, it was shown how to obtain improved Lagrange multiplier tests. This can be accomplished either by using corrected critical values from an asymptotic expansion of the null distribution of the Lagrange multiplier statistic or by applying a Bartlett-type correction to the test statistic.

(Submetido em maio de 1992. Revisado em outubro de 1992) 


\section{References}

Anderson, T.W. 1984. An Introduction to Multivariate Statistical Analysis, 2nd ed. New York: Wiley.

Attfield, C. L.F. 1991. "A Bartlett adjustment factor to the likelihood ratio test for homoskedasticity in the linear model." Economics Letters 37:119-123.

Barndorff-Nielsen, O.E. \& Cox, D.R. 1989. Asymptotic Techniques for Use in Statistics. London: Chapman and Hall.

Berndt, E. R. \& Savin, N.E. 1977. "Conflict among criteria for testing hypotheses in the multivariate linear regression model." Econometrica 45: 1263-1277.

Breusch, T.S. 1979. "Conflict among criteria for testing hypotheses: extensions and comments." Econometrica 47:203-207.

Breusch, T.S. \& Schmidt, P. 1988. "Alternative forms of the Wald test: how long is a piece of string?" Communications in Statistics, Theory and Methods 17:2789-2795.

Buse, A. 1982. "The likelihood ratio, Wald, and Lagrange multiplier tests: an expository note." The American Statistician 36: $153-157$.

Chandra, T.K. \& Joshi, S. N. 1983. "Comparison of the likelihood ratio, Rao's and Wald's tests and a conjecture of C.R. Rao." Sankhyā A 45: 226-246.

Cordeiro, G. M. C. 1993. "Bartlett corrections and bias correction for two heteroscedastic regression models." Communications in Statistics, Theory and Methods, forthcoming.

Cordeiro, G. M. \& Ferrari, S. L.P. 1991. "A modified score test having chi-squared distribution to order $n^{-1}$." Biometrika 78: 573-582.

Cordeiro, G.M., Ferrari, S. L.P. \& Paula, G. A. 1993. "Improved score tests for generalized linear models." Journal of the Royal Statistical Society B, forthcoming.

Cox, D. R. 1988. "Some aspects of conditional and asymptotic inference: a review." Sankhyā A 50:314-337.

Cribari-Neto, F. \& Ferrari, S.L.P: 1992. "An improved Lagrange multiplier statistic for the test of heteroskedasticity." Discussion 
Paper RT-MAE-9210, Department of Statistics, University of São Paulo.

Dagenais, M. G. \& Dufour, J. M. 1991. "Invariance, nonlinear models, and asymptotic tests." Econometrica 59: 1601-1615.

1992. "On the lack of invariance of some asymptotic tests to rescaling." Economics Letters 38:251-257.

Engle, R. 1984. "Wald, likelihood ratio, and Lagrange multiplier tests in econometrics." In Griliches, Z. \& Intriligator, M., org., Handbook of Econometrics. Amsterdam: North-Holland.

Evans, G.B.A. \& Savin, N.E. 1982. "Conflict among the criteria revisited: the W, LR and LM tests." Econometrica 50:737-748.

Ferrari, S.L.P. \& Cordeiro, G.M. 1990. "Fórmulas matriciais para o fator de correção da estatística escore." Revista Brasileira de Estatística 51:101-113.

Ferrari, S.L.P. \& Cordeiro, G.M. 1992. "Matrix formulae for computing improved score tests." Mimeo, Department of Economics, Federal University of Pernambuco.

Godfrey, L. G. 1978. "Testing for multiplicative heteroskedasticity." Journal of Econometrics 8:227-236.

1988. Misspecification Tests in Econometrics. Cambridge, MA: Cambridge University Press.

Gregory, A. W. \& Veall, M. R. 1985. "Formulating Wald tests of nonlinear restrictions." Econometrica 53:1465-1468.

Griffiths, W.E. \& Surekha, K. 1986. "A Monte Carlo evaluation of the power of some tests for heteroskedasticity." Journal of Econometrics 31:219-231.

Harris, P. 1985. "An asymptotic expansion for the null distribution of the efficient score statistic." Biometrika 72:653-659.

Hayakawa, T. \& Puri, M.L. 1985. "Asymptotic expansions of some test statistics." Annals of the Institute of Statistical Mathematics A 37:95-108.

Hill, G.W. \& Davis, A.W. 1968. "Generalized asymptotic expansions of Cornish-Fisher type." Annals of Mathematical Statistics 39: $1264-1273$.

Honda, Y. 1988. "A size correction to the Lagrange multiplier test for heteroskedasticity." Journal of Econometrics 38:375-386. 
Italianer, A. 1985. "A small-sample correction for the likelihood ratio test." Economics Letters 19:315-317.

Koenker, R. 1981. "A note on studentizing a test for heteroskedasticity." Journal of Econometrics 17:107-112.

Lafontaine, F. \& White, K.J. 1986. "Obtaining any Wald statistic you want." Economics Letters 21:35-40.

McCullagh, P. 1987. Tensor Methods in Statistics. London: Chapman and Hall.

Mizon, G. E. 1977. "Inferential procedures in nonlinear models: an application in a U.K. industrial cross-section study of factor substitution and returns to scale." Econometrica 45:1221-1242.

Mizon, G.E. \& Hendry, D.F. 1980. "An empirical application and Monte Carlo analysis of of tests of dynamic specification." Review of Economic Studies 47:21-45.

Mukerjee, R. 1992. "Parametric orthogonality and a Bartlett-type modification for Rao's statistic in the presence of a nuisance parameter." Statistics and Probability Letters 13:397-400.

Nagar, A. L. \& Chandrika, C. 1992. "Edgeworth approximations to the distributions of the likelihood ratio and $\mathrm{F}$ statistics in the null and non-null cases." In Bewley, R. \& Hoa, T. V., org., Contributions to Consumer Demand and Econometrics: Essays in Honor of Henri Theil. New York: St. Martin's Press.

Neyman, J. 1959. "Optimal asymptotic tests of composite statistical hypothesis." In Grenander, U., org., Probability and Statistics, the Harald Cramer Volume. New York: Wiley.

Phillips, P.C.B. \& Park, J.Y. 1988. "On the formulation of Wald tests of nonlinear restrictions." Econometrica 56:1065-1083.

Rao, C.R. 1947. "Large sample tests of statistical hypothesis concerning several parameters with applications to problems of estimation." Proceedings of the Cambridge Philosophical Society 44: 50-57.

Reid, N. 1988. "Saddlepoint methods and statistical inference." Statistical Science 3:213-238.

1991. "Approximations and asymptotics." In D. V. Hinkley, Reid, N. \& Snell, E. J., org., Statistical Theory and Modelling: In Honour of Sir David Cox, FRS. London: Chapman and Hall. 
Rothenberg, T. J. 1984. "Hypotheses testing in linear models when the covariance matrix is nonscalar." Econometrica 52:827-842. Savin, N.E. 1976. "Conflict among testing procedures in a linear regression model with autoregressive disturbances." Econometrica 44: 1303-1315.

Sims, C. 1980. "Macroeconomics and reality." Econometrica 48:148. Reprinted in Granger, C. W. J., org., Modelling Economic Series, Oxford: Oxford University Press, 1990.

Smith, R. 1987. "Alternative asymptotically optimal tests and their applications to dynamic specification." Review of Economic Studies 54:665-680.

Ullah, A. \& Zinde-Walsh, and W tests in regression models." Econometrica 52: 1055-1066. 1985. "Estimation and testing in a regression model with spherically symmetric errors." Economics Letters 17:127132.

Wald, A. 1943. "Tests of statistical hypothesis concerning several parameters when the number of observations is large." Transactions of the American Mathematical Society 54:426-482.

White, H. 1984. Asymptotic Theory for Econometricians. New York: Academic Press.

Wolak, F. A. 1987. "An exact test for multiple inequality and equality constaints in the linear regression model." Journal of the American Statistical Association 82:782-793. 